\title{
Accurate Modeling of Multilayer Packaging Structures with a Hybrid FDTD Method
}

\author{
Edan T. K. Dalton ${ }^{1}$, Marco Kunze ${ }^{2}$, Wolfgang Heinrich ${ }^{2}$, and Manos M. Tentzeris ${ }^{1}$ \\ ${ }^{1}$ Georgia Institute of Technology, Atlanta, GA-30332, United States \\ ${ }^{2}$ Ferdinand-Braun-Institut für Höchfrequenztechnik (FBH), D-12489 Berlin, Germany
}

\begin{abstract}
This paper presents a method of coupling a static electromagnetic field solver with the finite-difference timedomain method for the more efficient modeling of multilayer packaging structures. Lossy metal characteristics are first simulated with a dense static grid and the resulting field correction factors are then used to enhance the accuracy of a much coarser FDTD mesh.
\end{abstract}

\section{Introduction}

Significant attention is currently being devoted to the accurate modeling of packaged RF modules. Many of these structures involve multilayer dielectrics interspersed with layers of metallization. These metals can be modeled as perfect electronic conductors, but most of the time, the finite thickness and imperfect material characteristics play a major role in the performance of the real devices. Also, irregularities and structure details used in these geometries, including vias and wire bond connections, may be of a much smaller geometrical scale in comparison to the rest of the topologies.

The finite-difference time-domain (FDTD) technique [1] has been employed to model many types of RF structures due to its versatility in simulation of high-frequency structures. However, as device features become smaller and integration increases, the computational load on a simulator becomes much larger. Since the Yee cell size is determined by the smallest feature of the device, and the number of cells is related to the total size of the structure, the computational domain quickly becomes more and more unwieldy. With the addition of non-ideal material parameters and with the incorporation of the finite thickness and conductivity of metallization, the grid can become very large while the size of the time-step gets much smaller. When a thin finiteconductivity metal layer is modeled in FDTD, a very fine grid must be used in order to accurately portray the field variation in the area around and inside the metal layer, something that leads to an impractical simulation time. Thus, a realistic model of the structure becomes impractical for simulation because of the unrealistic time needed for the simulation. Static solutions have been considered as a subgridding method of reducing the computational overhead required for modeling discontinuous structures [2], and including imperfect material characteristics in the finitedifference method [3].

The formulation of a hybrid FDTD method integrating a quasistatic field solver is presented. This solver is first used to solve for potentials over a conducting plane, such as a metallization layer in a packaged microsystem. The output from the static solver can be used to determine the areas of highest field variation, and correction factors can be derived to account for inaccuracies caused by the use of a coarser dynamic (FDTD) grid.

\section{Hybrid Method Overview}

The combination of the static solver and the FDTD method is an attempt to resolve some of the difficulties associated with modeling highly conductive thin materials in the time domain. Researchers often rely on heuristic approaches to generate the mesh for full-wave simulation, and this can result in inaccuracies in areas of large field variation. The static solver is used in conjunction with a variable-gridded FDTD mesh to reduce errors in mesh size near structure discontinuities. The main advantage of the static solver is that, since it has no time-marching component, it does not take very long to process, compared to a dynamic simulation of comparable resolution. In addition, it can be used as a preprocessing step that can optimize the grid size of the full-wave simulation.

For the integration of the static solver with the dynamic FDTD solver, correction factors for the EM fields for areas of high field variation are calculated. They improve the accuracy of the coarser dynamic grid through the solution of the integral forms of Ampere's and Faraday's laws over a very fine static grid. The equations needed for the calculation of the correction factors are given in (1) and (2), where $C F_{l}$ represents the line integral correction factor, $C F_{A}$ represents the surface integral correction factor, and the $\Delta$-terms represent the sizes of the FDTD coarse cells around which the integrals are discretized using the quasistatic values. The $\mathrm{dl}, \mathrm{dx}$, and dy variables of integration are the quasistatic grid sizes, which are much smaller than the coarser FDTD cells. The $\mathrm{F}$ components in the numerators of the expressions are the field values derived from the quasistatic solver over the fine grid, and the F component in the denominator is the field value derived from the quasistatic solver in the same location as the field position on the FDTD coarse grid. The error can thus be reduced by using a very large number of static, finegrid cells per dynamic, coarse-grid cells.

$$
\begin{gathered}
C F_{l}^{F}=\frac{\int \vec{F} \cdot d \vec{l}}{\bar{F} \cdot \Delta l} \\
C F_{A}^{F}=\frac{\iint \vec{F} \cdot d \vec{x} \cdot d \vec{y}}{\bar{F} \cdot \Delta x \cdot \Delta y}
\end{gathered}
$$

Figure 1 shows a representation of the calculation for the surface integral correction factors. The coarse FDTD grid is displayed in the bolded blue dashed lines on the grid, and the fine static grid is shown by the fine black solid lines. In this 
case, the two grids are shown as uniform, with respect to both themselves and each other, but this does not have to be the case. The technique can be applied using variable gridding for both the fine and coarse grids. The red dot at the center of the coarse FDTD cell denotes the location of the field used to calculate the denominator of (1).

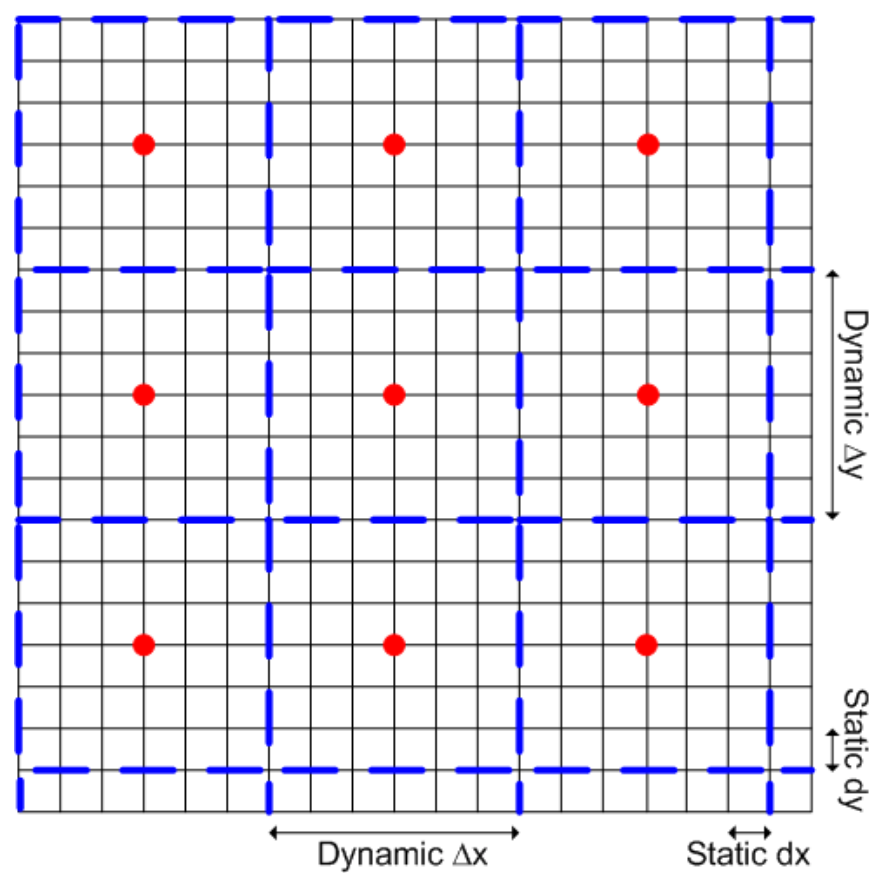

Figure 1: Grid showing relationship of dynamic and static cells.

The surface integral is calculated to a very high degree of accuracy using the static solver over the fine grid, and, as shown in (2), this expression is used as the numerator for the correction factor calculation. The denominator is determined by taking the field value at the center of the dynamic grid and multiplying it by the area of the FDTD cell. These calculations are carried out over the entire grid, and are performed before the dynamic simulation, thus avoiding additional computational overhead. In areas of large field variation, the magnitude of the correction factors can be quite large.

Another method we may use for the calculation of the correction factors, besides the quasistatic field solver, is a 2.5D FDTD [4] simulation with a very dense, high-resolution grid. This essentially provides the fine-grain field solution needed for the more accurate approximation of the integral equation solution around the areas of high field variation. The correction factors can then be calculated in the same manner as described above, with only the source of the field values being different between the two cases.

Following the derivation of the FDTD update equations from the integral forms [1] and incorporating the correction factor terms in our formulation, we derive the update expressions for $H_{x}$ and $E_{x}$ as shown in (3) and (4) (conductivity terms and nonessential subscripts are omitted for simplicity):

$$
\begin{aligned}
& \left.E_{x}\right|_{j, k} ^{n+.5}=\left.E_{x}\right|_{j, k} ^{n-.5}+\frac{2 \Delta t}{\left.\varepsilon \cdot C F_{A}^{E x}\right|_{j, k}} . \\
& \left(\left.\left.C F_{l}^{H y}\right|_{j+.5, k} \cdot H_{y}\right|_{j+.5, k} ^{n}-\left.\left.C F_{l}^{H y}\right|_{j-.5, k} \cdot H_{y}\right|_{j-.5, k} ^{n}\right. \\
& \Delta y \\
& \left(\frac{\left.\left.C F_{l}^{H z}\right|_{j, k+.5} \cdot H_{z}\right|_{j, k+.5} ^{n}-\left.\left.C F_{l}^{H z}\right|_{j, k-.5} \cdot H_{z}\right|_{j, k-.5} ^{n}}{\Delta z}\right) \\
& \left.H_{x}\right|_{j-.5, k-.5} ^{n+.5}=\left.H_{x}\right|_{j-.5, k-.5} ^{n-.5}+\frac{2 \Delta t}{\left.\mu \cdot C F_{A}^{H x}\right|_{j-.5, k-.5}} .
\end{aligned}
$$

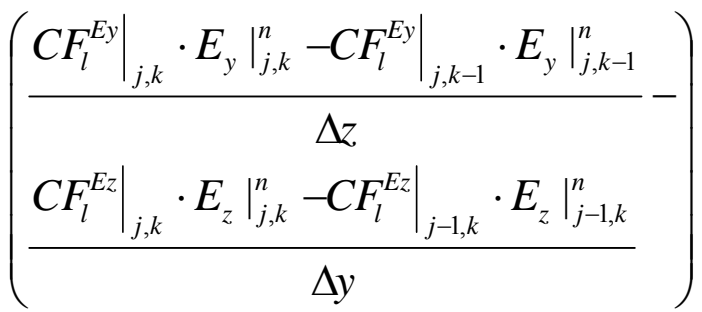

The relationship of the field components and the approximations of the integral equations is shown in Figure 1 , which displays the contours and surfaces used in this calculation. The contour integral of the electric field is related to the surface integral of the magnetic field by Faraday's law. In this figure, the solved-for field is $\mathrm{H}_{\mathrm{z}}$, related to the loop including $\mathrm{E}_{\mathrm{x}}$ and $\mathrm{E}_{\mathrm{y}}$. Equation (4) can be derived in a similar way.

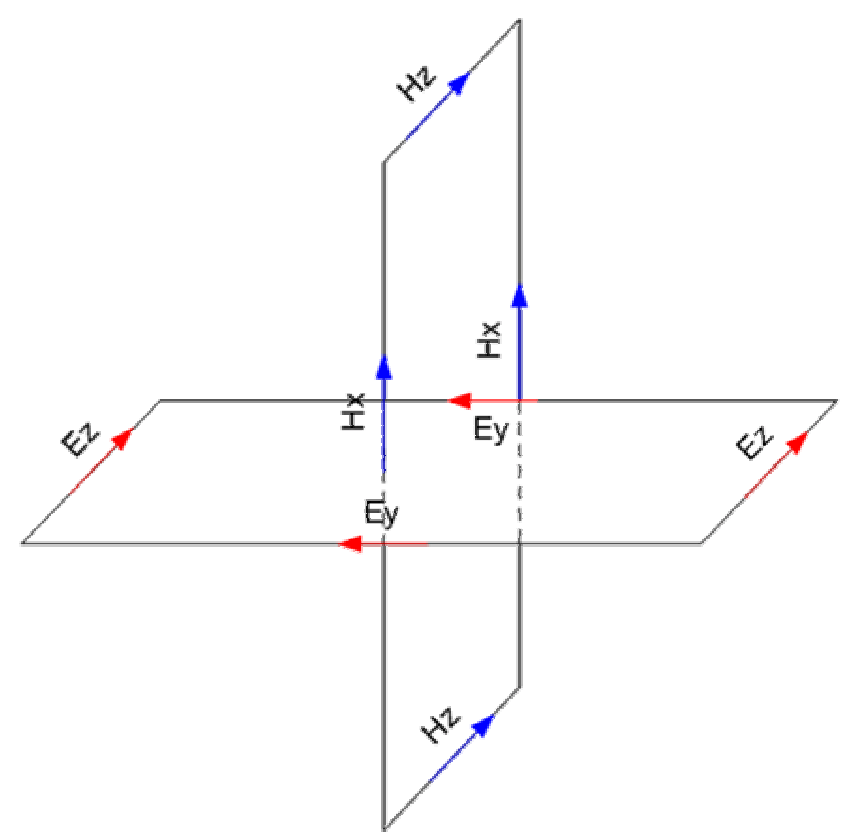

Figure 2: Faraday contour showing relation of fields for the calculation of $\mathrm{H}_{\mathrm{x}}$ in (4).

\section{Preliminary Results}

The above-described technique was benchmarked for a simple microstrip structure with thin finite-conductivity metal strips. Some preliminary results derived from these simulations are described below. 
Figure 3 illustrates the areas where the correction factors are significantly applicable and shows the basic geometry for the test structure, a microstrip line on polyimide $\left(\varepsilon_{\mathrm{r}}=3.2\right)$ with a gold metallization $\left(\sigma=3 \times 10^{7} \mathrm{~S} / \mathrm{m}\right)$. The substrate thickness is $20 \mu \mathrm{m}$, with a strip height of $5 \mu \mathrm{m}$. Initially, the quasistatic solver was used for the whole structure and the dashed area was derived after thresholding the correction factor values and neglecting those in areas of low field variation.

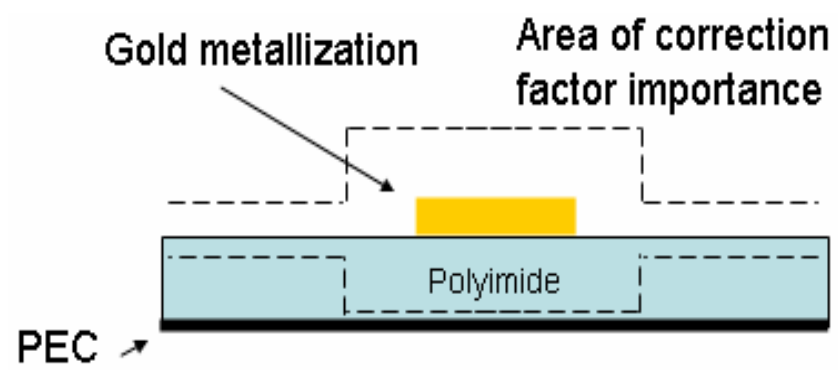

Figure 3: Test structure geometry with indicated area of correction factor application

The structure was first simulated using a high-resolution FDTD grid, in order to determine the field distributions in a very dense grid. The field distribution for the high-resolution grid is shown in Figure 4. The high-resolution grid is 94 by 95 by 100 cells, with a minimum cell size of $200 \mathrm{~nm}$, and uses 10 cells of PML in all directions. It employs 25 cells for the thickness of the $5 \mu \mathrm{m}$ strip, and 20 cells for the $42 \mu \mathrm{m}$ strip width. The resulting simulation was extremely computationally intensive and required more than a day to run.

The same structure was then modeled with the quasistatic solver deriving the correction factors and for the development of the FDTD grid. The coarse dynamic grid, which utilizes one Yee cell (in one dimension) for a $5 \mu \mathrm{m}$ metallization, was on average one-half to one-third (in two dimensions) the resolution of the fine static grid. The static grid is 47 by 70 by 27 cells, and the FDTD simulation uses a grid of 22 by 23 by 27 cells, with 8 cells of PML above the structure, and 4 around its perimeter. In Figure 4, the area of the strip is magnified due to the high resolution of the grid in this area. The coarse grid and the correction factors were then applied to the simulation of the structure in FDTD. The field distribution for the coarse grid incorporating correction factors is shown in Figure 5. The larger cell size contributes to the appearance of a more spread out field distribution in the figure. As can be seen from the figures, the areas of high field variation are extremely difficult to simulate using coarse grids, but the correction factors tend to reduce the error.

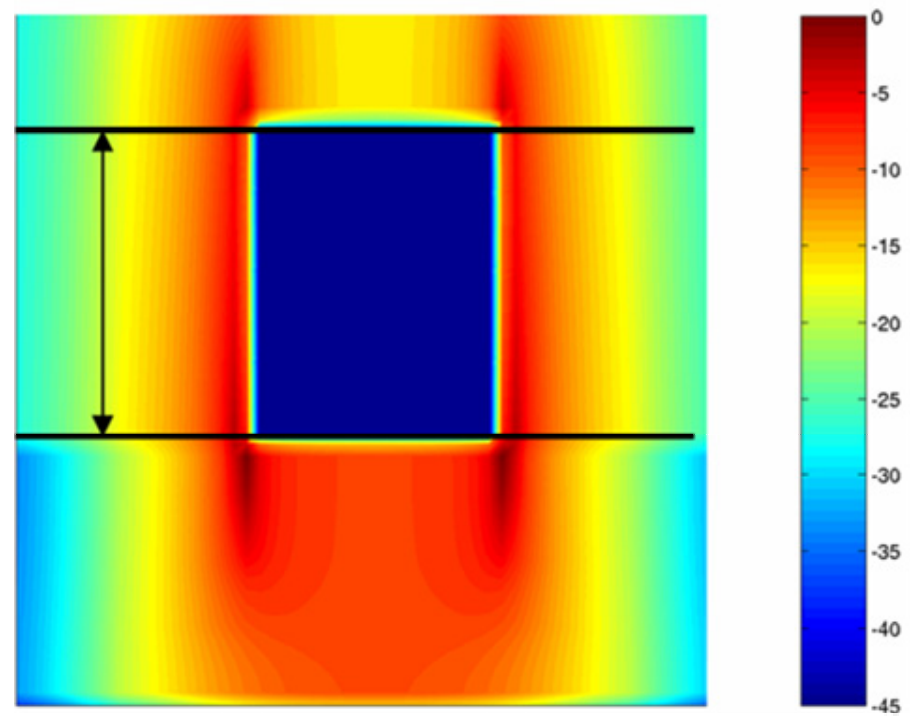

Figure 4: Electric field distribution derived from highresolution FDTD simulation (area between lines has been enlarged-strip height is actually $5 \mu \mathrm{m}$ ).

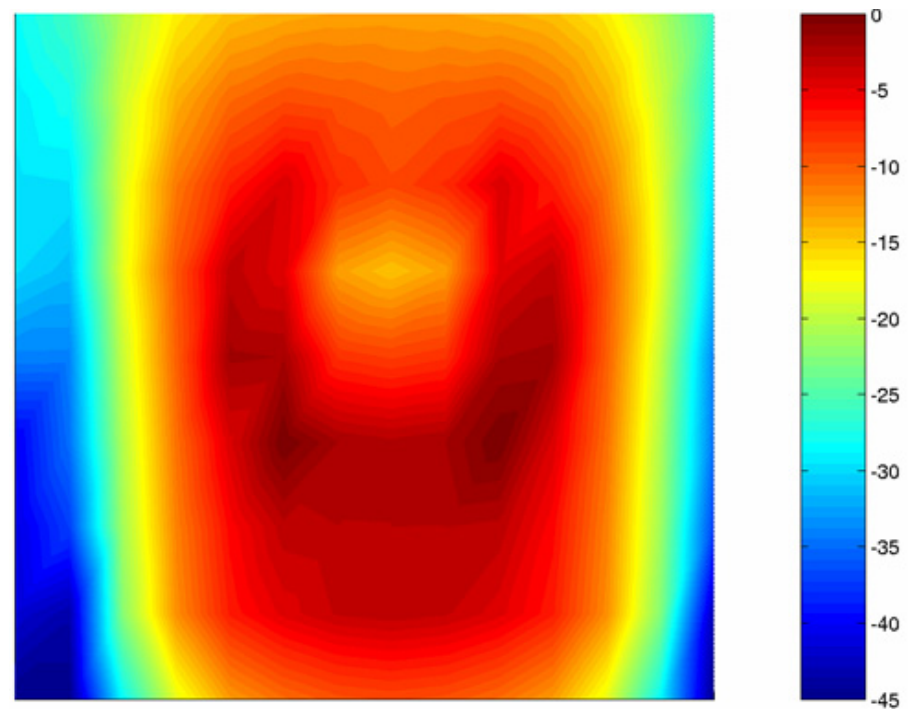

Figure 5: Electric field distribution from coarse grid using correction factors

\section{Conclusion}

In this paper, a method of coupling a static simulator with a conventional FDTD code for the purpose of improving accuracy while reducing simulation time through "subgridding" is presented. This is accomplished by the use of correction factors in the FDTD equations that can be derived from the quasistatic analysis of RF structures over a much finer grid. This technique can also be used to eliminate "guesswork" in meshing by determining areas of highest field variation from the magnitudes of the correction factors, something very important in the simulation of modern complex integrated RF modules or smart antenna arrays.

The high resolution FDTD grid gives good results, but due to its large number of cells, it is a very computationally intensive simulation. For the purposes of this research, the structure was gridded with a total of just under one million cells. This in itself is not such a heavy load, but the 
computational situation was compounded by the necessarily small space step which then resulted in an extraordinarily small time step. As a result, the simulation needed over 400,000 time steps to converge, or approximately a day and a half, using an in-house developed parallel FDTD code on a 14 processor workstation cluster segment. This is the sort of situation it is felt that the hybrid technique presented here can be useful. By comparison, the static solver and coarse FDTD simulation take approximately an hour combined to simulate the test structure.

\section{Acknowledgement}

The authors wish to acknowledge the assistance of the Packaging Research Center at Georgia Tech, the Yamacraw Research Initiative of the State of Georgia, and NSF Career award \#9984761.

\section{References}

1. A. Taflove and S. C. Hagness, Computational Electrodynamics: The Finite-Difference Time-Domain Method, pp.109-142, Artech House, Boston, 2000.

2. D. B. Shorthouse and C.J. Railton, "The incorporation of static field solutions into the finite difference time domain algorithm," IEEE Trans on Microwave Theory and Techniques, Vol. 40, pp. 986-994, May 1992.

3. M. Kunze and W. Heinrich, "3D hybrid finite-difference method for lossy structures based on quasi-static field solutions," Proc. of the 2002 IEEE IMS, pp. 1881-1884, Seattle, WA, June 2002.

4 S. Xiao, R Vahldieck, and H. Jin, "Full-wave analysis of guided wave structures using a novel 2-D FDTD," IEEE Microwave and Guided Wave Letters, pp 165-167, May 1992. 